\title{
Design of Compact Symmetric Four-Port Crossover Junction
}

\author{
Yi-Chyun Chiou, Member, IEEE, Jen-Tsai Kuo, Senior Member, IEEE, and Hsuan-Rong Lee
}

\begin{abstract}
Performance of a fully planar four-port crossover coupler is investigated. This bisymmetric circuit consists of a ring and two inner orthogonal sections connecting the diagonal ports. Based on the transmission line theory, analysis equations are formulated and design curves are presented. Two crossover junctions are fabricated and measured for validity confirmation. The measured data have good agreement with simulation.
\end{abstract}

Index Terms-Crossover, directional coupler, four-port network, transmission line theory.

\section{INTRODUCTION}

$\mathbf{M}$ ICROSTRIP crossing is one of the fundamental discontinuities in microwave and millimeter-wave integrated circuits [1]-[3]. When two transmission lines cross over each other, an intuitive way to isolate the signals on them is to employ three-dimensional structures, e.g., bond wires or air bridges [2], [3]. These elements will definitely increase the fabrication cost. It is noted that a series of multiport coupler circuits in a fully planar configuration have been developed for the crossover applications [4]-[9]. An ideal four-port crossover provides $0 \mathrm{~dB}$ insertion loss to diagonal ports and perfect isolation to adjacent ports. In [4], microstrip and stripline crossovers are designed based on a cascade of two branch-line hybrids. In [5], octavewide matched symmetrical and reciprocal four- and five-port networks are investigated. In [6], a circular disk is proposed for a symmetrical and reciprocal four-port crossover junction. In [7], a novel planar configuration of $0 \mathrm{~dB}$ directional coupler is presented as a single-layer crossover in microwave integrated circuits. The "window"-shape circuits in [8] are composed of several branch-line couplers connected in two dimensions. Such circuits may have an improved bandwidth if the characteristic impedances of the constitution branches can be properly designed. Recently, in [9], a double-ring structure is proposed for design of microstrip four-port crossover coupler. The eigenmode model is developed to investigate the circuit performance. The measured data show that the isolation and return losses of the prototype coupler exceed $20 \mathrm{~dB}$ over a bandwidth of $20 \%$.

In this letter, the symmetric four-port crossover junction in [5] is analyzed and its performance is investigated. It deserves

Manuscript received March 05, 2009; revised May 20, 2009. First published August 11, 2009; current version published September 02, 2009. This work was supported in part by the MoE ATU program and by the National Science Council, Taiwan, under Grants NSC 97-2221-E-009-039 and NSC 98-2218-E009-011.

The authors are with the Department of Communication Engineering, National Chiao Tung University, Hsinchu 300, Taiwan. (e-mail: jtkuo@ mail.nctu. edu.tw).

Color versions of one or more of the figures in this paper are available online at http://ieeexplore.ieee.org.

Digital Object Identifier 10.1109/LMWC.2009.2027054

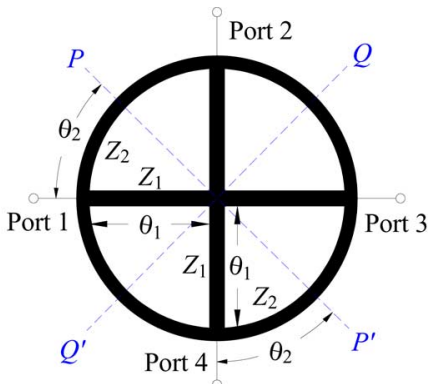

(a)

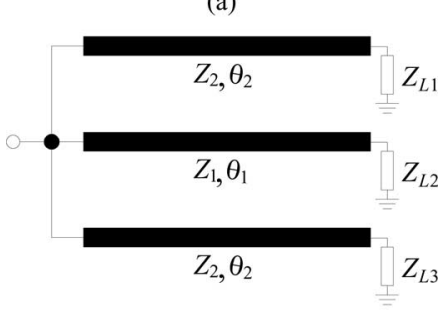

(b)

Fig. 1. Crossover coupler under analysis. (a) Circuit layout. (b) Reduced transmission line circuit in formulation.

further investigation since only several discrete sets of solutions, but neither simulation nor measured results, are shown in [5] and its circuit structure, and hence its analysis, is much simpler than the double-ring configuration in [9]. Design graphs are generated based on formulation by the transmission line theory, and simulated circuit bandwidths of the solutions are discussed. Two circuits are fabricated and measured for confirmation.

\section{ANALYSIS}

Fig. 1(a) shows the layout of the crossover coupler under analysis, where $P P^{\prime}$ and $Q Q$ ' are planes of symmetry. Let the characteristic impedance and the electric length of each section (from center to port) be $Z_{1}$ and $\theta_{1}$, respectively, and the counterparts of the entire ring peripheral be $Z_{2}$ and $8 \theta_{2}$. Since the structure possesses bisymmetry, $P P^{\prime}$ and $Q Q^{\prime}$ can be assigned as either electric or magnetic wall for ease of analysis. When this is done, the reduced network becomes a one-port network shown as in Fig. 1(b). The load impedances $Z_{L i}(i=1,2,3)$ are either zero or infinite depending on electric or magnetic wall is applied. Four reflection coefficients can be derived and all $S$-parameters of the junction can be obtained [10]. When input is excited at port 1, for instance, $S_{11}=S_{21}=S_{41}=0$ and $\left|S_{31}\right|=1$ at the design frequency are required. It leads to $\Gamma_{m m}=\Gamma_{e e}=-\Gamma_{e m}=-\Gamma_{m e}$ and $\left|\Gamma_{m m}\right|=\left|\Gamma_{e e}\right|=$ $\left|\Gamma_{e m}\right|=\left|\Gamma_{m e}\right|=1$, where the subscripts $e$ and $m$ stand for that $P P^{\prime}$ and $Q Q$ ' planes are placed with electric and magnetic 


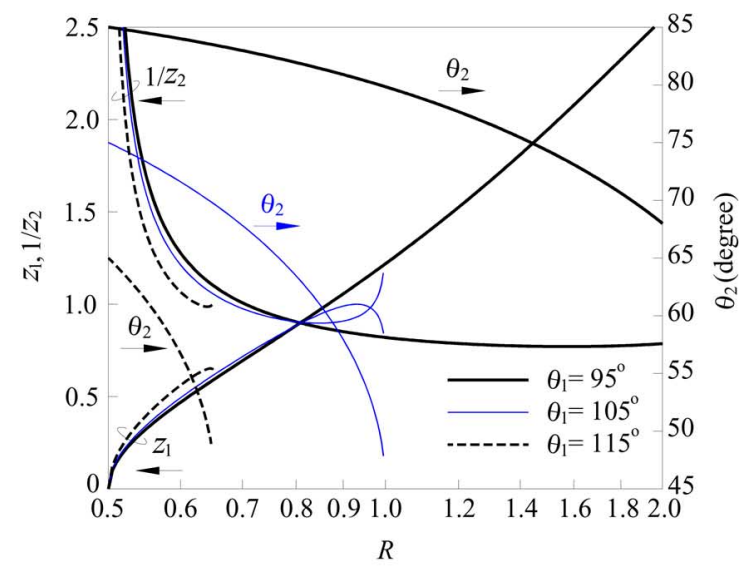

(a)

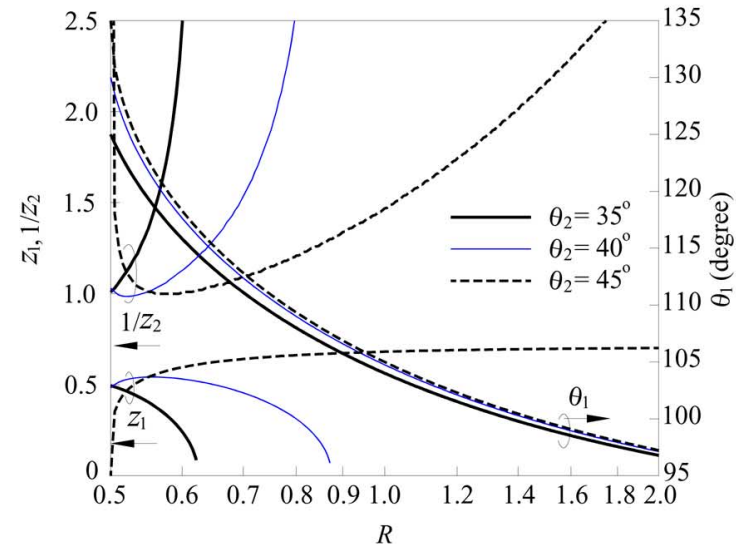

(b)

Fig. 2. $z_{1}, 1 / z_{2}$ and $\theta_{k}$ as functions of $R$. (a) $k=2$. (b) $k=1$.

walls, respectively. Consequently, the following two equations can be derived:

$$
\begin{aligned}
s_{2} c_{2}+2 R s_{1} c_{1} & =0 \\
c_{2}^{2}\left[c_{1}^{2}\left(3-t_{2}^{2}\right)-c_{2}^{2}\left(1-t_{2}^{2}\right)\right] & =2 c_{1}^{2}\left(z_{1}^{2} s_{1}^{2}+c_{1}^{2}\right)
\end{aligned}
$$

where $R=Z_{1} / Z_{2}, z_{k}=Z_{k} / Z_{o}(k=1$ and 2$), Z_{o}$ is reference impedance, $t_{k}=\tan \theta_{k}, s_{k}=\sin \theta_{k}$, and $c_{k}=\cos \theta_{k}$. These two equations are used to solve the four circuit parameters $z_{1}, z_{2}, \theta_{1}$ and $\theta_{2}$. Here, $\theta_{k}$ denotes electric length of the corresponding section at the design frequency.

Fig. 2(a) plots $z_{1}, 1 / z_{2}$ and $\theta_{2}$ as functions of $R$ (in log scale) given that $\theta_{1}=95^{\circ}, 105^{\circ}$ and $115^{\circ}$. When $\theta_{1}=105^{\circ}$ and $115^{\circ}$, and when $R$ is larger than 1 and 0.655 , respectively, $\theta_{2}$ has no real solutions. When $\theta_{1}=95^{\circ}, z_{1}$ and $1 / z_{2}$ solutions cover 0.5 $\leq R \leq 2.0$. Fig. 2(b) plots $z_{1}, z_{2}$ and $\theta_{1}$ as functions of $R$ for $\bar{\theta}_{2}=\overline{3} 5^{\circ}, 40^{\circ}$ and $45^{\circ}$.

\section{Simulation AND Measurement}

The solutions in Fig. 2 possess different circuit performances. Tables I and II compare the simulation bandwidths of some solutions sampled at $R=0.6,0.8(0.85)$ and 1.8 in Figs. 2(a) and (b), respectively. The bandwidths are measured using $\left|S_{11}\right|<$ $-20 \mathrm{~dB},\left|S_{21}\right|<-20 \mathrm{~dB}$, and $\left|S_{31}\right|>-0.5 \mathrm{~dB}$. It is found that many results in Table I have a bandwidth less than $1 \%$. To have the three bandwidths of about $10 \%$ or more, Table II suggests $\theta_{2}$
TABLE I

CiRCUIT BANDwidTHS (\%) OF SAMPLED SOLUTIONS IN Fig. 2(A)

\begin{tabular}{|c|c|c|c|c|}
\hline \hline$R$ & $\theta_{1}$ & $\left|S_{11}\right|<-20 \mathrm{~dB}$ & $\left|S_{21}\right|<-20 \mathrm{~dB}$ & $\left|S_{31}\right|>-0.5 \mathrm{~dB}$ \\
\hline \multirow{3}{*}{0.6} & $95^{\circ}$ & 0.45 & 0.40 & 0.10 \\
\cline { 2 - 5 } & $105^{\circ}$ & 0.48 & 0.57 & 1.09 \\
\cline { 2 - 5 } & $115^{\circ}$ & 1.95 & 2.92 & 5.35 \\
\hline \multirow{2}{*}{0.8} & $95^{\circ}$ & 0.13 & 0.13 & 0.29 \\
\cline { 2 - 5 } & $105^{\circ}$ & 2.62 & 2.50 & 2.13 \\
\hline 1.8 & $95^{\circ}$ & 0.47 & 0.47 & 0.97 \\
\hline \hline
\end{tabular}

TABLE II

Circuit BANDWidThS (\%) OF SAMPLED SOlutions IN Fig. 2(B)

\begin{tabular}{|c|c|c|c|c|}
\hline \hline$R$ & $\theta_{2}$ & $\left|S_{11}\right|<-20 \mathrm{~dB}$ & $\left|S_{21}\right|<-20 \mathrm{~dB}$ & $\left|S_{31}\right|>-0.5 \mathrm{~dB}$ \\
\hline \multirow{3}{*}{0.6} & $35^{\circ}$ & 1.29 & 65.53 & 4.57 \\
\cline { 2 - 5 } & $40^{\circ}$ & 10.98 & 18.17 & 15.66 \\
\cline { 2 - 5 } & $45^{\circ}$ & 10.85 & 9.95 & 16.98 \\
\hline \multirow{2}{*}{0.85} & $40^{\circ}$ & 0.84 & 77.38 & 2.97 \\
\cline { 2 - 5 } & $45^{\circ}$ & 9.08 & 9.88 & 12.86 \\
\hline 1.8 & $45^{\circ}$ & 2.90 & 15.74 & 7.80 \\
\hline \hline
\end{tabular}

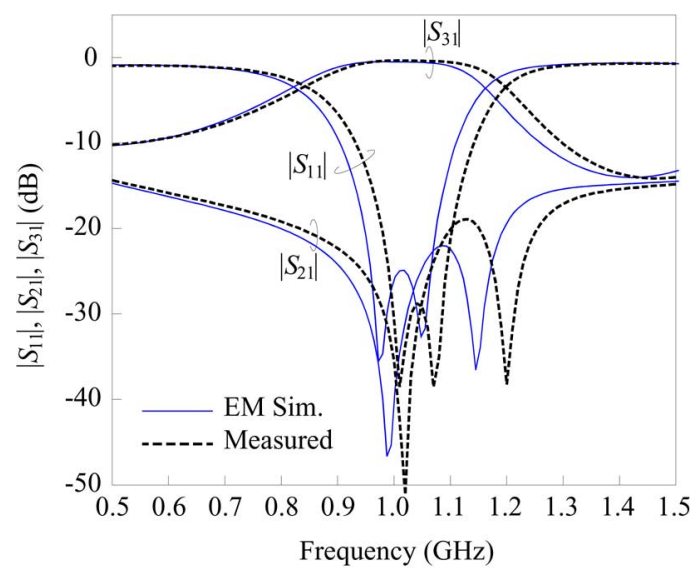

(a)

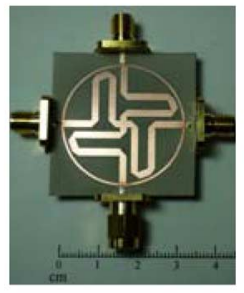

(b)

Fig. 3. (a) Magnitudes of the $S$-parameters. (b) Photograph of the measured crossover. $Z_{1}=26.23 \Omega, Z_{2}=50.8 \Omega(R=0.52), \theta_{1}=125^{\circ}, \theta_{2}=38^{\circ}$.

be close to $40^{\circ} \sim 45^{\circ}$ and $R$ close to $0.5 \sim 0.6$. However, both $z_{1}$ and $z_{2}$ may be far beyond the fabrication limit of the standard PCB process when $R<0.55$. Based on the simulation results (not shown), the maximum bandwidth of the proposed structure is approximately $20 \%$. If wider bandwidths are required, optimization could be inevitable.

Two circuits designed at $f_{o}=1 \mathrm{GHz}$ are fabricated and measured for demonstration. The circuit substrate has a relative dielectric constant $\varepsilon_{r}=10.2$ and thickness $=0.635 \mathrm{~mm}$. Fig. 3(a) plots the simulated and measured results of the first circuit. The circuit dimensions are listed in the caption. Note that 


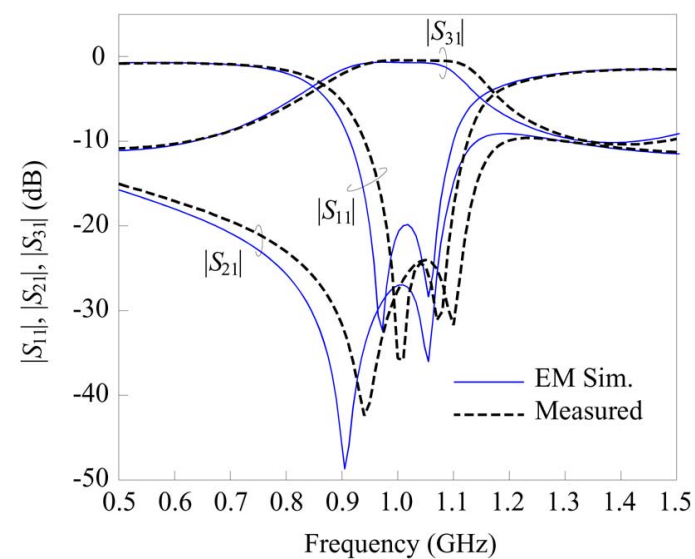

Fig. 4. Performance of the second coupler. $Z_{1}=25.06 \Omega, Z_{2}=$ $55.2 \Omega(R=0.45), \theta_{1}=132^{\circ}, \theta_{2}=32.5^{\circ}$.

$\theta_{1}$ is larger than the radius of the ring, i.e., $8 \theta_{2} / 2 \pi$, the inner sections then have to be folded to accommodate the area inside the ring. Circuit simulation is done by the software package IE3D [11]. At the design frequency, the measured data show that $\left|S_{11}\right|,\left|S_{21}\right|=\left|S_{41}\right|$ and $\left|S_{31}\right|$ are $-31.6 \mathrm{~dB},-50.5 \mathrm{~dB}$ and $-0.385 \mathrm{~dB}$, respectively, and that the total power loss $\left(P_{L}=\right.$ $\left.1-\left|S_{11}\right|^{2}-2\left|S_{21}\right|^{2}-\left|S_{31}\right|^{2}\right)$ is $8.48 \%$. The $20 \mathrm{~dB}$ bandwidths of $\left|S_{11}\right|$ and $\left|S_{21}\right|$ are $12.3 \%$ and $25.6 \%$, respectively, and the $0.5 \mathrm{~dB}$ bandwidth of $\left|S_{31}\right|$ is $13 \%$. One can see that the measured results agree well with the simulation data. It is worth mentioning that this circuit occupies about $25 \%$ of the area of the double-ring structure in [9]. Fig. 3(b) shows the photograph of the measured coupler.

Fig. 4 plots the simulated and measured results of the second crossover junction. The measured return loss and isolation at $f_{o}$ are $26.4 \mathrm{~dB}$ and $24.9 \mathrm{~dB}$, respectively, and coupling between ports 1 and 3 is $0.455 \mathrm{~dB}$. In comparison with the previous coupler, the $20 \mathrm{~dB}$ isolation bandwidth is larger, while the return loss and insertion loss are more or less the same. The circuit area is $73 \%$ of that of the previous one.

\section{CONCLUSION}

Performance of a symmetric four-port crossover coupler is investigated. Compared to the circuit reported recently in [9], it has a much simpler configuration and analysis equations. The design graphs show that the designated crossover function can be achieved with versatile circuit dimensions and performances. Two circuits with different areas are fabricated and measured for verification. Both circuits show good insertion loss between diagonal ports and excellent isolation between adjacent ports.

\section{REFERENCES}

[1] S.-C. Wu, H.-Y. Yang, N. Alexopoulos, and I. Wolff, "A rigorous dispersive characterization and microstrip cross and T junctions," IEEE Trans. Microw. Theory Tech., vol. 38, no. 12, pp. 1837-1844, Dec. 1990.

[2] G. E. Ponchak and E. Tentzeris, "Development of finite ground coplanar (FGC) waveguide 90 degree crossover junctions with low coupling," in IEEE MTT-S Int. Dig., Jun. 2000, pp. 1891-1894.

[3] T.-S. Hong, "A rigorous study of microstrip crossovers and their possible improvements," IEEE Trans. Microw. Theory Tech., vol. 42, no. 9, pp. 1802-1806, Sep. 1994.

[4] J. S. Wight, W. J. Chudobiak, and V. Makios, "A microstrip and stripline crossover structure," IEEE Trans. Microw. Theory Tech., vol. MTT-24, no. 5, p. 270, May 1976.

[5] F. C. de Ronde, "Octave-wide matched symmetrical, reciprocal, 4- and 5 ports," in IEEE MTT-S Int. Dig., Jun. 1982, pp. 521-523.

[6] K. C. Gupta and M. D. Abouzahra, "Analysis and design of four-port and five-port microstrip disk circuits," IEEE Trans. Microw. Theory Tech., vol. MTT-33, no. 12, pp. 1422-1428, Dec. 1985.

[7] D. V. Kholodniok and I. Vendik, "A novel type of 0-dB directional coupler for microwave integrated circuits," in Proc. 29th Eur. Microw. Conf., Nov. 1999, pp. 341-344.

[8] D. V. Kholodniok, G. Kalinin, E. Vernoslova, and I. Vendik, "Wideband 0-dB branch-line directional couplers," in IEEE MTT-S Int. Dig., Jun. 2000, pp. 1307-1310.

[9] Y. Chen and S.-P. Yeo, "A symmetrical four-port microstrip coupler for crossover application," IEEE Trans. Microw. Theory Tech., vol. 55 , no. 11, pp. 2434-2438, Nov. 2007.

[10] R. E. Collin, Foundations for Microwave Engineering, 2nd ed. Singapore: McGraw-Hill, 1992.

[11] IE3D Simulator. Fremont, CA: Zeland Software, Inc., Jan. 1997. 